\title{
Impact of relatively new chemotherapeutic agents on the outcome of Egyptian patients with advanced malignant pleural mesothelioma
}

\author{
Gaafar R, Khaled H, Sallam YA*, Shafik H, Aboulkassem FA, Emara M \\ Medical Oncology Department, NCl, Cairo University, Egypt
}

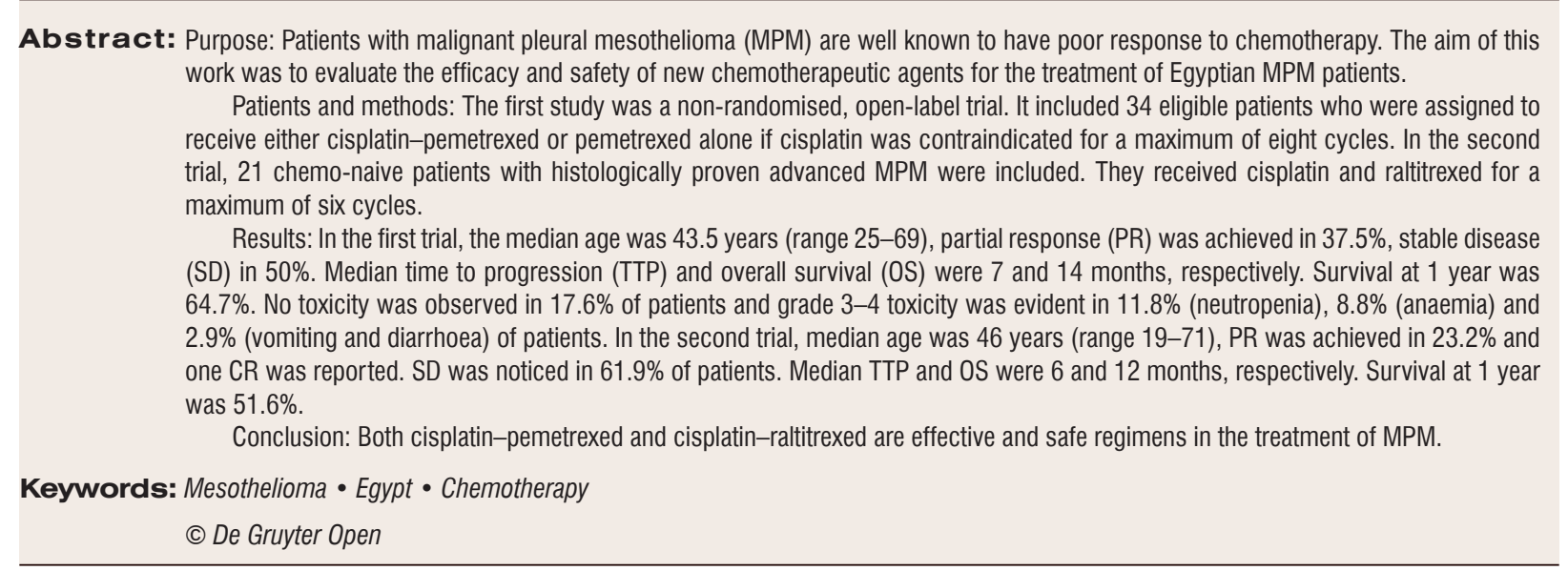

\section{Introduction}

Malignant pleural mesothelioma (MPM) is an increasing problem in Egypt. More than 1000 employees work on the asbestos lines and are consequently exposed to the material [1]. Mesothelioma in Egypt is mainly attributed to environmental source with a high incidence in women and young adults. Epidemiological data for 635 malignant mesothelioma patients over 4 years in the third millennium were collected from the National Cancer Institute $(\mathrm{NCl})$, Cairo University, and Abbassia Chest hospital, Cairo (both hospitals train and serve most of the high risk population living in the neighbourhoods of asbestos factories). The median age was 53 (19-90) years, and male to female ratio 1:5. Residential exposure was evident in $64.7 \%$ [1]. In a study conducted at $\mathrm{NCl}$, Cairo, on 100 (MPM) patients, asbestos exposure was reported in $67 \%$ of cases as they were residents in endemic areas. Median time of exposure was 36 years. SV40 (simian virus 40) was detected in $60 \%$ of the cases [2].

MPM is a locally invasive and rapidly fatal malignancy. Surgical resection is possible in a minority of patients. For those who are not treated with curative resection, fewer than $15 \%$ live beyond 5 years. The median survival time of 337 patients in 11 multicentre chemotherapy trials was reported to be 7 months [3]; however, systemic chemotherapy remains one of the few therapeutic options that has been proven in a randomised controlled trial to improve survival in patients with stages I-IV medically inoperable MPM and as part of a regimen for patients with medically operable MPM, and those with sarcomatoid histology [4].

Of all novel agents tested for the treatment of MPM, none achieved single-agent activity of more than $20 \%$ 
[5-10]. In a thorough systematic search of the literature for published articles and abstracts, cisplatin was found to be the most active single-agent with a response rate $20 \%$ [11]. The European Society of Medical Oncology (ESMO) guidelines recommend pemetrexed-cisplatin or raltitrexed-cisplatin as first-line treatment for advanced mesothelioma.

Pemetrexed, a multitargeted antifolate, has shown modest activity as a single-agent in a phase II trial of patients with MPM RR 14.1\% [12]. A large, phase III clinical trial that included 456 advanced chemotherapynaive patients were randomly assigned to receive pemetrexed $500 \mathrm{mg} / \mathrm{m}^{2}$ and cisplatin $75 \mathrm{mg} / \mathrm{m}^{2}$ on day 1 , or cisplatin $75 \mathrm{mg} / \mathrm{m}^{2}$ on day 1 every 21 days. The results of this study showed that the median survival time in the pemetrexed-cisplatin arm was 12.1 versus 9.3 months in the control arm ( $p=0.02$, two-sided logrank test). The hazard ratio for death of patients in the pemetrexed-cisplatin arm versus those in the control arm was 0.77 . Median time to progression (TTP) was significantly longer in the pemetrexed-cisplatin arm: 5.7 versus 3.9 months $(p=0.001)$. RR was $41.3 \%$ in the pemetrexed-cisplatin arm versus $16.7 \%$ in the control arm $(p<0.0001)$. After 117 patients had been enrolled, folic acid and vitamin $B_{12}$ were added to reduce toxicity, resulting in a significant reduction in toxicities in the pemetrexed-cisplatin arm. It was concluded that pemetrexed-cisplatin therapy was associated with superior survival duration compared with cisplatin alone [13].

Another promising drug is raltitrexed (Tomudex), which is a quinazoline folate analogue that acts as a pure and specific thymidine synthase inhibitor. A phase II study with single-agent raltitrexed in unpretreated patients showed an RR of $21 \%$ [14]. Further studies consisting of raltitrexed combined with oxaliplatin have demonstrated response rates varying from $20 \%$ to $35 \%$ in patients with MPM [15].

So, a second large phase III trial of the European Organisation for Research and Treatment of Cancer (EORTC) (08983) that included 250 patients was done and demonstrated that cisplatin and raltitrexed significantly improved median survival compared with single-agent cisplatin (11.4 versus 8.8 months; hazard ratio $0.76, p=0.0483)$. Overall, $R R(24 \%$ versus $14 \%, p$ $=0.056$ ) was greater in the combination treatment arm, but this difference was not statistically significant [16].

\section{Patients and methods}

Over a period of 2 years, patients with histologically proven MPM who were not candidates for curative surgery received at the $\mathrm{NCl}$, Cairo, outpatient clinic were assessed for eligibility to get enrolled in one of two trials on pemetrexed and raltitrexed. Both studies were exploring two drugs that proved effective for better response and survival compared with other treatments used for advanced and metastatic.

Eligibility requirements included uni- or bidimensionally measurable disease, age $\geq 18$ years with life expectancy $\geq 12$ weeks, a WHO performance status of $0, \mathrm{I}$ or II and adequate hepatic and renal function and bone marrow reserve; baseline values had to be at least: haemoglobin, $10 \mathrm{~g} / \mathrm{dL}$; white blood cell count, $4 \times 109 / \mathrm{L}$; absolute neutrophil count, $2 \times 109 / \mathrm{L}$; thrombocyte count, $100 \times 109 / \mathrm{L}$; albumin, $3 \mathrm{~g} / \mathrm{dL}$; and creatinine clearance (measured or calculated), $65 \mathrm{~mL} / \mathrm{min}$. Bilirubin had to be lower than $1.46 \mathrm{mg} / \mathrm{dL}$ and serum creatinine lower than $1.69 \mathrm{mg} / \mathrm{dL}$. Alanine aminotransferase and aspartate aminotransferase had to be lower than $2.5 \times$ the upper normal limit, except in the case of liver involvement, where it had to be less than $5 \times$ the upper normal limit. Pulmonary function tests were not routinely done. Patients were excluded if they had a second primary malignancy or brain metastases.

Patients who received pemetrexed containing regimen were excluded if they were unable to interrupt non-steroidal anti-inflammatory drugs (NSAID) 2 days before, the day of and 2 days after pemetrexed (longacting NSAID should be interrupted 8 days before pemetrexed). A written informed consent was essential.

Pemetrexed-cisplatin regimen was given to chemonaive patients and for patients previously treated on neoadjuvant, adjuvant or metastatic setting with a cisplatin based regimen who responded for $\geq 6$ months, and who had no medical contraindications to receiving additional cisplatin, such as grade $3 / 4$ neuropathy or ototoxicity.

Single-agent pemetrexed was given to patients with medical contraindications for receiving additional cisplatin such as grade $3 / 4$ neuropathy or ototoxicity. This was a non-randomised, open-label trial; patients were assigned to a regimen per investigators' clinical decision. Pemetrexed was administered intravenously (IV) at $500 \mathrm{mg} / \mathrm{m}^{2}$ over 10 minutes, on day 1 of each 21-day cycle for a maximum of eight cycles; for combination, pemetrexed administration was followed 30 minutes later by cisplatin $75 \mathrm{mg} / \mathrm{m}^{2} \mathrm{IV}$ over 2 hours. Pre- and post-hydration was administered according to local practice; dexamethasone, $4 \mathrm{mg}$ or equivalent corticosteroid orally twice per day on the day before, the day of, and the day after each dose of pemetrexed. Folic acid 350-600 ug was taken orally daily beginning 1-2 weeks before the first chemotherapy doses and was continued throughout study therapy. Vitamin $B_{12}$ 1000 ug was given intramuscularly $1-2$ weeks before 
the first dose of study therapy and repeated every 9 weeks until study discontinuation to reduce the risk of severe skin rash.

Other chemotherapy, immunotherapy or hormonal therapy was not permitted. Supportive care therapies were allowed during the study. Dose adjustments for haematological toxicity were based on a stepwise reduction schedule. Grade 3 or 4 mucositis, diarrhoea requiring hospitalisation, or grade 3 or 4 nonhaematologic effects also resulted in dose reduction for subsequent doses. Any patient requiring three dose reductions was discontinued from the study. Dose delays up to 42 days were permitted for recovery from study drug toxicity. Dose escalations were not allowed.

For patients who received raltitrexed, received in combination with cisplatin. In all, 21 patients were included in this study. They received cisplatin and raltitrexed. Palliative radiotherapy for painful lesions or for preventing the development of metastases along biopsy tracks was allowed. Patients had to provide written informed consent before randomisation. The primary outcome was overall survival (OS). Secondary outcomes included Progression-free Survival (PFS), safety and RR.

Raltitrexed was administered IV at $3 \mathrm{mg} / \mathrm{m}^{2}$ for 15 minutes, preceding cisplatin. Both drugs were given on day 1 of each cycle and repeated every 3 weeks until progression, severe toxicity or patient refusal. Standard intravenous hydration and antiemetic regimens were given. Prophylactic administration of any vitamin or colony stimulating factor was not recommended. Dose adjustments of raltitrexed in the next treatment cycles were recommended for any combination of diarrhoea and haematological toxicity according to the following schedule: raltitrexed $2.25 \mathrm{mg} / \mathrm{m}^{2}$ for any grade 2 diarrhoea and for any grade 3 haematological toxicity; and raltitrexed $1.5 \mathrm{mg} / \mathrm{m}^{2}$ for any grade 3 diarrhoea and/or grade 4 haematological toxicity. Raltitrexed was withdrawn in case of any grade 4 diarrhoea and if a grade 3 diarrhoea coincided with a grade 3 haematological toxicity. A patient discontinued protocol treatment if grade 3 or 4 toxicity occurred after a dose reduction or if three dose reductions were required. Dose delays up to 14 days were permitted for recovery from study drug toxicity. Dose escalations were not allowed.

Baseline and pre-dosing assessment in both trials included a complete history and physical examination, complete blood cell count, calculated creatinine clearance, liver enzymes, blood electrolytes, blood albumin, calcium and glucose, and vitamin metabolites.

OS was defined as the time from randomisation to the time of death from any cause. Patients who were alive on the date of last follow-up were censored on that date. TTP was defined as the time from randomisation until documented progression or death from any cause. For patients without DP at the time of analysis, the date of last follow-up was considered right-censored. Duration of tumour response was defined as the time from the first objective status of a response to the time of documented DP or death from any cause. Time to treatment failure was defined as the time from randomisation to the date of observed DP, death from any cause or early discontinuation of treatment.

Chest imaging was performed at least once just before every other treatment while a patient was receiving study therapy and approximately every 6 weeks after completion of study therapy.

Response to treatment was assessed by measuring the thickness of up to three involved areas of pleura at each of three separate levels at least $2 \mathrm{~cm}$ apart on computed tomography scan, at baseline and every other cycle (at least one measurement was $\geq 1.5 \mathrm{~cm}$ ). Complete remission (CR) was defined as complete absence ofmeasurable, non-measurable butassessable, and unassessable disease with no new lesions and no disease-related symptoms. A partial response (PR) was defined as $\geq 30 \%$ decrease in the sum of the greatest diameters of unidimensionally measurable lesions. Any $\mathrm{CR}$ or $\mathrm{PR}$ required confirmation 4 weeks later. Tumour response rate was defined as the percent of patients who experienced either a CR or PR.

Tumour progression was defined as the appearance of a new or relapsed lesion/site, $a \geq 20 \%$ increase in the sum of the longest dimension of unidimensionally measurable lesions over smallest sum. Stable disease (SD) was disease that did not qualify for CR, PR or progression during therapy.

\section{Statistical analysis}

The end points of both trials were response rate, OS, PFS and toxicity (graded according to the Common Toxicity Criteria). Statistical analysis was performed using SPSS version 14. Descriptive statistics was presented as frequency tables, means and standard deviations whenever appropriate. OS and PFS were estimated on all patients using the Kaplan-Meier analysis.

Unless otherwise noted, all tests of hypotheses were conducted at the alpha of 0.05 level, with a $95 \%$ confidence interval.

\section{Results}

Thirty-four patients received pemetrexed containing regimen; $19 \%$ were stage I, $33.4 \%$ stage III and $47.6 \%$ stage IV. 
In all, $29(85.3 \%)$ received pemetrexed-cisplatin, and $5(14.7 \%)$ received pemetrexed alone. Median time from diagnosis to starting treatment was 2.5 months (9 days-24.5 months). The total number of cycles for all patients was 199, with a median of six cycles/ patient range (2-8) cycles. Patients' characteristics are described in table (1). Overall, $50 \%$ of the patients received previous therapy; $8.8 \%$ (three patients) were subjected to surgery in the form of EEP or decortication, and only one patient received palliative radiotherapy and $13(38.3 \%)$ received combination chemotherapy

Table 1. Patients charactaristics in Pemetrexed study.

\begin{tabular}{c|c}
\hline Characteristic & No (\%) \\
\hline Sex & $21(61.8 \%)$ \\
Male & $13(38.2 \%)$ \\
female & 43.5 \\
Median age (range), years & $(25-69)$ \\
PS & \\
II & $22(64.7 \%)$ \\
I & $12(35.3 \%)$ \\
Asbestos exposure & $20(58.8 \%)$ \\
Prior therapy & $17(50 \%)$ \\
Surgery(EPP or decortication) & $3(8.8 \%)$ \\
Palliative radiotherapy & $1(2.9 \%)$ \\
chemotherapy & $13(38.3 \%)$ \\
Pathology & \\
Epithelial & $20(58.8 \%)$ \\
Mixed & $7(20.6 \%)$ \\
Sarcomatoid & $6(17.6 \%)$ \\
other & $1(3.0 \%)$ \\
Site & \\
Pleural & $31(91.2 \%)$ \\
peritoneal & $3(8.8 \%)$ \\
\hline
\end{tabular}

(cisplatin-gemcitabin). The pleura was the primary disease site in $91.2 \%$ of patients and peritoneum in $8.8 \%$ of patient.

None of the patients achieved CR, and 12 patients $(37.5 \%)$ achieved PR. SD was noted in 16 patients $(50 \%)$. All patients were followed till death. Median OS was 13 months $(95 \% \mathrm{Cl}, 12$ to 14$)$. Survival at 12 and 18 months was $64.7 \%$ and $23.5 \%$, respectively (Fig (1)). There was a statistically significant difference in OS between patients with PS 1 and those with PS > $1, p$ value $=0.002$ (Fig (2)); however, this difference was not noted between patients with epithelial histology and other histology. Median TTP was 7 months $(95 \% \mathrm{Cl}, 2.8$ to 11.1) (Fig (3)). There was no statistically significant difference in TTP between patients with PS 1 and PS > $1(p=0.16)$, or epithelial histology compared with other histologies $(p=0.51)$. Haematological toxicity in the form of neutropenia was the most commonly encountered and diarrhoea was the commonest non-haematological toxicity (table (2)). None of the patients received secondline chemotherapy; upon progression, they received either radiotherapy or best supportive care.

Twenty-one patients received raltitrexed-cisplatin; $3.4 \%$ were stage II, $48.3 \%$ stage III and $48.3 \%$ stage IV. Median interval between first diagnosis and randomisation was 52 days. Total number of cycles for all patients was 110 , with a median of six cycles/patient range (2-8) cycles. Patients' characteristics are shown in table (3).

One patient reached CR and five patients $(23.8 \%)$ achieved PR. SD was noted in 13 patients $(61.9 \%)$. Median survival for all patients was 12 months $(95 \% \mathrm{Cl}$, 9 to 15) (Fig (4)). There was no statistically significant difference in OS between patients with PS 1 and those

Table 2. Toxicity of pemetrexed containing regimen.

\begin{tabular}{c|cccc}
\hline Toxicity, n(\%) & Grade 1 & Grade 2 & Grade 3 & Grade 4 \\
\hline \hline Neutropenia & $1(2.9 \%)$ & $4(11.8 \%)$ & $2(5.9 \%)$ & $2(5.9 \%)$ \\
anemia & $1(2.9 \%)$ & $4(11.9 \%)$ & $3(8.8 \%)$ & 0 \\
thrombocytopenia & 0 & $1(2.9 \%)$ & $1(2.9 \%)$ & 0 \\
diarrhea & $1(2.9 \%)$ & 0 & $2(5.9 \%)$ & 0 \\
vomiting & $2(5.9 \%)$ & $2(5.9 \%)$ & $1(2.9 \%)$ & 0 \\
renal & 0 & $1(2.9 \%)$ & $1(2.9 \%)$ & 0 \\
Elevated liver enzymes & 0 & 0 & 0 & 0 \\
mucositis & $3(8.8 \%)$ & 0 & 0 & 0 \\
neuropathy & $3(8.8 \%)$ & 0 & 0 & 0 \\
allergy & $3(8.8 \%)$ & 0 & 0 \\
\hline
\end{tabular}


Table 3. Patients characteristics for Raltitrexed study.

\begin{tabular}{c|c}
\hline Characteristic & No (\%) \\
\hline \hline Sex & $15(71.4 \%)$ \\
Male & $6(28.6 \%)$ \\
female & 46.1 \\
Median age (range), years & $(19-71)$ \\
PS & \\
I & $12(57.1 \%)$ \\
I & $9(42.9 \%)$ \\
Asbestos exposure & $16(76.2 \%)$ \\
Pathology & \\
Epithelial & $16(76.2 \%)$ \\
Mixed & $4(19 \%)$ \\
unknown & $1(4.8)$ \\
\hline
\end{tabular}

Table 4. Toxicity of raltetrexed containing regimen.

\begin{tabular}{|c|c|c|c|c|}
\hline $\begin{array}{c}\text { Grade } \\
4\end{array}$ & $\begin{array}{c}\text { Grade } \\
3\end{array}$ & $\begin{array}{c}\text { Grade } \\
2\end{array}$ & $\begin{array}{c}\text { Grade } \\
1\end{array}$ & Toxicity, n (\%) \\
\hline 0 & 0 & $10(47.6 \%)$ & $10(47.6 \%)$ & anorexia \\
\hline 0 & 0 & 7 (33.3\%) & $8(38.1 \%)$ & nausea \\
\hline 0 & 0 & 9 (42.9\%) & 10 (47.6\%) & vomiting \\
\hline 0 & 0 & $1(4.8 \%)$ & $2(9.5 \%)$ & diarrhea \\
\hline 0 & 0 & $1(4.8 \%)$ & $3(14.3 \%)$ & stomatitis \\
\hline 0 & 0 & $2(9.5 \%)$ & $2(9.5 \%)$ & alopecia \\
\hline 0 & 0 & $8(38.1 \%)$ & $10(47.6 \%)$ & fatigue \\
\hline 0 & 0 & 0 & $8(38.1 \%)$ & neurological \\
\hline 0 & 0 & 0 & $3(14.5 \%)$ & cardiovascular \\
\hline 0 & 0 & 0 & $1(4.8 \%)$ & allergy \\
\hline 0 & 0 & 0 & $1(4.8 \%)$ & $\begin{array}{c}\text { Febrile } \\
\text { neutropenia }\end{array}$ \\
\hline 0 & 0 & 0 & 0 & anemia \\
\hline 0 & 0 & 0 & 0 & thrombocytopenia \\
\hline 0 & 0 & 0 & 0 & renal \\
\hline 0 & 0 & 0 & 0 & $\begin{array}{l}\text { Elevated liver } \\
\text { enzymes }\end{array}$ \\
\hline
\end{tabular}

with PS > $1(p=0.23)$, and epithelial histology and other histology $(p=0.44)$. Median TTP was 6 months (95\% Cl, 2 to 10) (Fig (5)). Haematological toxicity was not remarkable. Anorexia was the commonest non-haematological toxicity (table (4)). Second-line chemotherapy was given in $32 \%$ of patients.

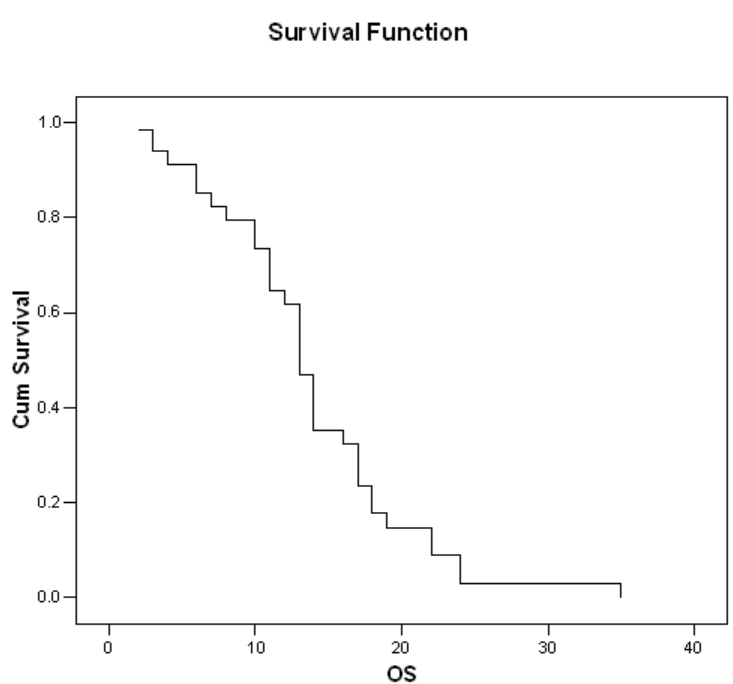

Figure 1. Overall survival of patients in pemetrexed study.

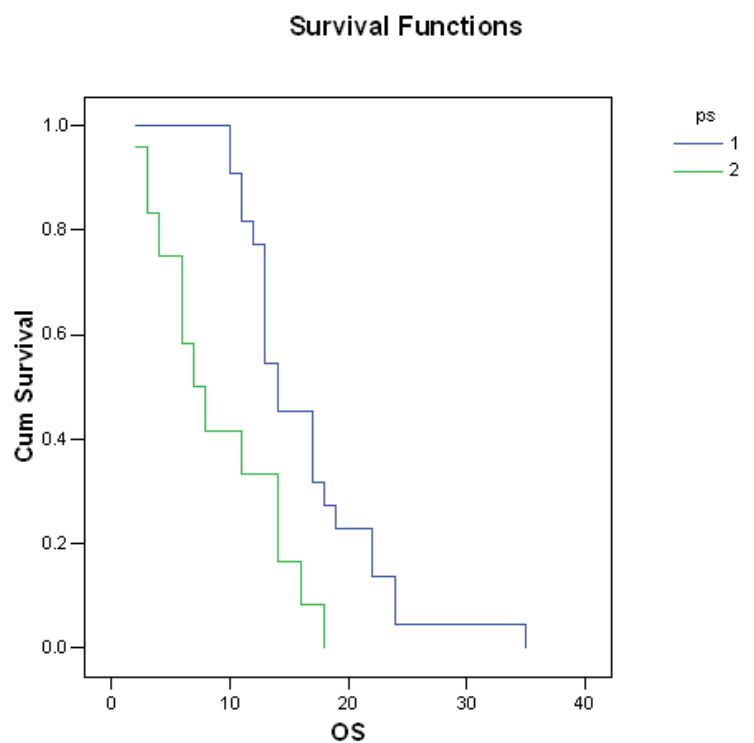

Figure 2. Comparison of OS in patients according to PS.

\section{Discussion}

In our first trial, 29 patients $(85.3 \%)$ received pemetrexedcisplatin and five patients $(14.7 \%)$ received pemetrexed alone. Nineteen patients received this treatment as firstline, while 15 received it as second-line. None of the patients reached CR, 12 patients (37.5\%) achieved PR. $\mathrm{SD}$ disease was noted in 16 patients $(50 \%)$. Median TTP was 7 months $(95 \% \mathrm{Cl}, 2.8$ to 11.1$)$. The median survival of our patients was 13 months $(95 \% \mathrm{Cl} 12$ to 14).

Our data are consistent with the data reported from a multicentre phase III study conducted on chemo-naive 


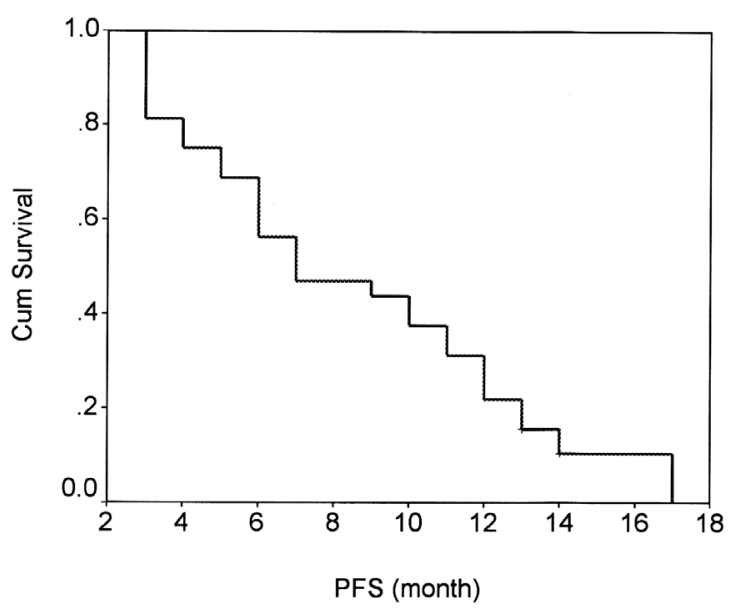

Figure 3. TTP in patients receiving pemetrexed containing regimen.

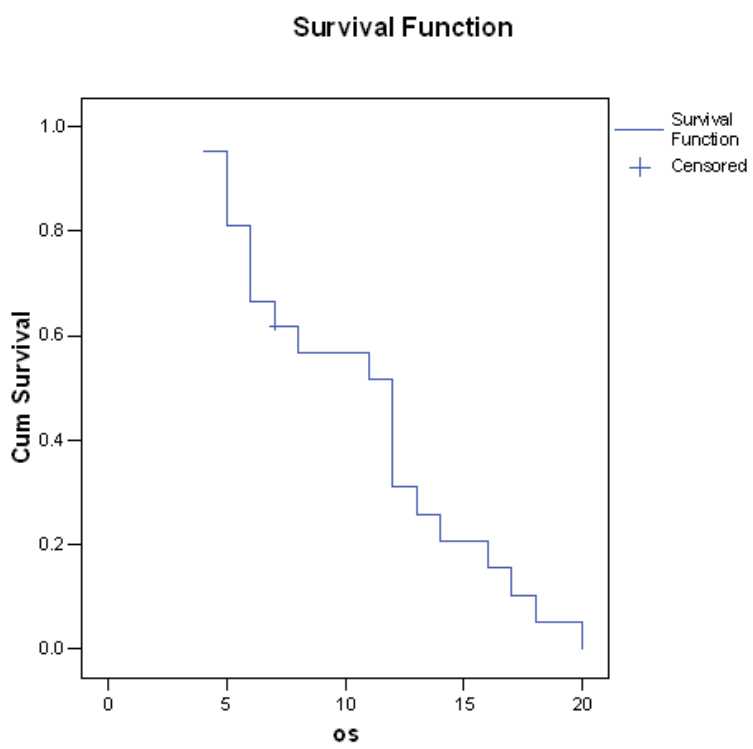

Figure 4. OS for patients receiving raltitrexed combination treatment.

patients with MPM who were not eligible for curative surgery where patients were randomised to receive either pemetrexed and cisplatin or cisplatin alone. RRs were $41.3 \%$ in pemetrexed-cisplatin arm, and $16.7 \%$ in cisplatin only arm. TTP was 5.7 and 3.9 months in both arms, respectively. There was a statistically significant higher median survival in pemetrexed-cisplatin arm versus cisplatin only (12.1 versus 9.3 months, $p=0.02$ ) [13].

Our data were also consistent with data reported by Castagneto et al. on 76 patients with measurable advanced MPM received pemetrexed $500 \mathrm{mg} / \mathrm{m}^{2}$ in combination with carboplatin AUC 5 every 21 days. Overall response rate was 25\%. In all, 39\% patients reported SD. The median survival was 14 months [17],

\section{Survival Function}

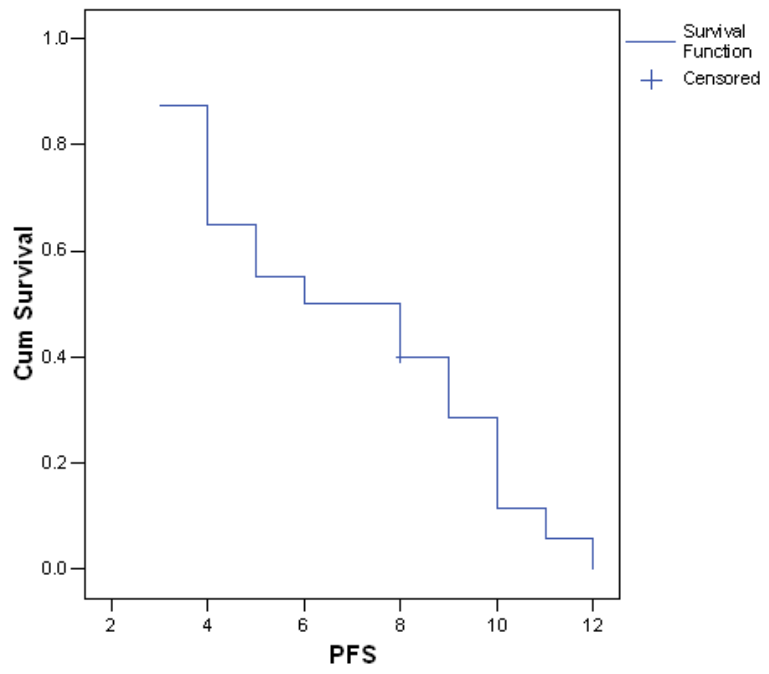

Figure 5. PFS for patients receiving raltitrexed containing regimen.

and for those reported by Ceresoli et al. on 102 patients, response rate of $18.6 \%$ was reached, and $47 \%$ had SD after treatment. Median TTP was 6.5 months; median OS was 12.7 months [18].

In a study on 1704 chemo-naive patients, the patients received pemetrexed plus cisplatin or pemetrexed plus carboplatin and were evaluated for safety. The pemetrexed plus cisplatin group demonstrated a response rate of $26.3 \%$ compared with $21.7 \%$ for the pemetrexed plus carboplatin group, with similar 1-year survival rates $(63.1 \%$ versus $64.0 \%)$ and median time to progressive disease (7 versus 6.9 months). The most common grade 3/4 haematologic toxicity was neutropenia in $23.9 \%$ of the pemetrexed plus cisplatin group and $36.1 \%$ of the pemetrexed plus carboplatin group [19]. These results were also consistent with our data.

Twenty-one patients received raltitrexed-cisplatin. One patient reached $\mathrm{CR}$, five patients $(23.8 \%)$ achieved PR. SD was noted in 13 patients (61.9\%). Median survival for all patients was 12 months $(95 \% \mathrm{Cl}, 9$ to 15). There was no statistically significant difference in OS between patients with PS 1 and those with PS > 1 $(p=0.23)$, and epithelial histology and other histology ( $p$ $=0.44)$. Median TTP was 6 months $(95 \% \mathrm{Cl}, 2$ to 10$)$. Haematological toxicity was not remarkable. Anorexia was the commonest non-haematological toxicity.

These data are consistent with those reported by Van Meerbeeck et al. When chemo-naive patients were randomised to receive cisplatin alone or raltitrexed and cisplatin, the RR was $13.6 \%$, and $23.6 \%$ in both arms, respectively $(p=0.056)$. The median OS was 8.8 months 
(95\% Cl, 7.8 to 10.8$)$ in cisplatin alone arm versus 11.4 months $(95 \% \mathrm{Cl}, 10.1$ to 15$)$ in raltitrexed, cisplatin arm $p=0.048$. Haematological toxicity was mild and consisted mostly of neutropenia. Grades 3 and 4 neutropenia occurred in $8 \%$ of patients receiving cisplatin alone and in $16 \%$ of patients receiving raltitrexed and cisplatin. Non-haematological toxicity was variable and mild, and no unexpected toxicities occurred [16].

In conclusion: The results of our trials using these drugs in Egyptian patients confirm the fact that both cisplatin-pemetrexed with vitamin supplementation and cisplatin-raltitrexed are effective and safe regimens in the treatment of MPM.

A combined first-line regimen using cisplatin with pemetrexed is considered the gold standard for MPM and is currently the only regimen approved by the US FDA for MPM, based on a phase III study, which assessed cisplatin-pemetrexed versus cisplatin alone in patients who were not candidates for surgery; the

\section{References}

[1] Gaafar R, Aly Ely Eldin N; Epidemic of mesothelioma in Egypt, Lung Cancer, 2005, 49S1,S17-S20.

[2] Abou El-Kasem F,Gaafar R, Abdel-Rahman A,Abdel-Baki H, Hassan N. Malignant pleural mesothelioma : analysis of 100 consecutive patients. Lung Cancer 2004, 45(3):s67.

[3] Porret E, Madelaine J, Galateau-Salle F, et al. Epidemiology, molecular biology, diagnostic and therapeutic strategy of malignant pleural mesothelioma in 2007-an update. Rev Mal Respir 2007; 24:6S157-64. [PubMed]

[4] Van Schil PE, Baas P, Gaafar R, et al. Trimodality therapy for malignant pleural mesothelioma: results from an EORTC phase II multicentre trial. Eur Respir J 2010;36:1362-9.

[5] Hillerdal G, Sorensen JB, Sundstrom S, et al. Treatment of malignant pleural mesothelioma with carboplatin, liposomized doxorubicin, and gemcitabine: a phase II study. J Thorac Oncol 2008; 3:1325-

[6] van Meerbeeck JP, Baas P, Debruyne C, et al: EORTC Lung Cancer Group. A phase II EORTC study of temozolomide in patients with malignant pleural mesothelioma. Eur J Cancer 2002, 38, 779-783.

[7] Habib EE, Fahmy ES. Chemotherapy management of malignant pleural mesothelioma: a phase II study comparing two popular chemotherapy regimens. Clin Transl Oncol. 2013 [Epub ahead of print] [PubMed]

[8] van Meerbeeck J, Debruyne C, van Zandwijk N, combination regimen increased survival in comparison with cisplatin alone (12.1 versus 9.3 months, $p=0.02$ ).

Agents inhibiting the Vascular Endothelial Growth Factor (VEGF) receptor, sunitinib, cedirinib and semaxanib, often in combination with other targets, have shown some evidence of activity in an unselected population, with response rates around $10 \%$ and PFS between 3 and 4 months. However, identifying predictors of benefit from these agents has proven difficult, with no predictive factors identified in extensive serum testing of the VEGF pathway in one recent study of sunitinib [20]. The anti-VEGF antibody bevacizumab has been tested in a number of completed and ongoing trials, with the best evidence coming from a randomised phase II trial of bevacizumab in combination with cisplatin and pemetrexed chemotherapy that did not show improved PFS or OS [21], although subset analyses suggested that a low serum VEGF level may predict improved outcomes on the combination [22].

et al: Paclitaxel for malignant pleural mesothelioma: A phase II studies of the EORTC Lung Cancer Cooperative Group. Br J Cancer, 1996, 74, 961963.

[9] Scherpereel A, Astoul P, Baas P, et al. Guidelines of the European Respiratory Society and the European Society of Thoracic Surgeons for the management of malignant pleural mesothelioma. Eur Respir J 2010; 35: 479-95.

[10] Sahmoud T, Postmus PE, van Pottelsberghe C, et al: G. Etoposide in malignant pleural mesothelioma: Two phase II trials of the EORTC Lung Cancer Cooperative Group. Eur J Cancer ,1997, 33, 2211-2115.

[11] Ellis P, Davies AM, Evans WK, etal: The use of chemotherapy in patients with advanced malignant pleural mesothelioma: a systemic review and practice guide lines. J Thoracic Oncol, 2006, 1(6), 591-601.

[12] Shin DM, Scagliotti GV, Kindler HL, et al: A phase II trial of pemetrexed in malignant pleural mesothelioma (MPM) patients: Clinical outcome, role of vitamin supplementation, respiratory symptoms and lung function. Proc Am Soc Clin Oncol, 2002, 21, 294 (abstr 1175).

[13] Vogelzang N , Rusthoven J., Symanowski J.,etal phase III Study of Pemetrexed in Combination With CisplatinVersus Cisplatin Alone in Patients With Malignant Pleural Mesothelioma, J Clin Oncol 2003, 21, 2636-2644.

[14] Baas P, Ardizzoni A, Grossi F, et al: EORTC Lung 
Cancer Group: The activity of raltitrexed (Tomudex) in malignant pleural mesothelioma: An EORTC phase II study (08992). Eur J Cancer 2003, 39, 353-357.

[15] Fizazi K, Doubre $H$, Le Chevalier $T$, et al: Combination of raltitrexed and oxaliplatin is an active regimen in malignant mesothelioma: Results of a phase II study. J Clin Oncol 2003, 21, 349- 354.

[16] van Meerbeeck JP, Gaafar R, Manegold C, et al. Randomized phase Illstudy of cisplatin with or without raltitrexed in patients with malignant pleural mesothelioma: an intergroup study of the European Organisation for Research and Treatment of Cancer Lung Cancer Group and the National Cancer Institute of Canada. J Clin Oncol 2005;23 : 6881-9.

[17] Ceresoli GL, Zucali PA, Favaretto AG, et al. Phase II study of pemetrexed plus carboplatin in malignant pleural mesothelioma. J Clin Oncol 2006; 24:1443.

[18] Castagneto B, Botta M, Aitini E, et al. Phase II study of pemetrexed in combination with carboplatin in patients with malignant pleural mesothelioma (MPM). Ann Oncol 2008; 19:370.
[19] Santoro A, O'Brien ME, Stahel RA, et al. Pemetrexed plus cisplatin or pemetrexed plus carboplatin for chemonaïve patients with malignant pleural mesothelioma: results of the International Expanded Access Program. J Thorac Oncol 2008; 3:756.

[20] Laurie SA, Gupta A, Chu Q, et al. Brief report: a phase II study of sunitinib in malignant pleural mesothelioma. The NCIC Clinical Trials Group. J Thorac Oncol 2011; 6:1950-4.

[21] Kindler HL, Karrison TG, Gandara DR, et al. Multicenter, double-blind, placebo-controlled, randomized phase II trial of gemcitabine/cisplatin plus bevacizumab or placebo in patients with malignant mesothelioma. J Clin Oncol 2012; 30:2509-15.

[22] Zucali PA, Ceresoli GL, De Vincenzo F, et al. Advances in the biology of malignant pleural mesothelioma. Cancer Treat Rev 201; 37:543-58. [PubMed] 